MS44-P08

\section{Beamline P02.1: a workhorse for high- resolution powder diffraction \& total scattering experiments at PETRA III, DESY}

Michael Wharmby ${ }^{1}$, Martin Etter ${ }^{1}$, Jozef Bednarcik ${ }^{1}$, Jo-Chi Tseng ${ }^{1}$,

Mario Wendt ${ }^{1}$, Sergej Wenz ${ }^{1}$

1. P02.1, PETRA III, DESY, Hamburg, Germany

email: michael.wharmby@desy.de

Powder diffraction is a standard tool for characterisation in a wide range of fields across the physical sciences: from materials science and metallurgy, to chemistry and solid state physics. Third generation synchrotron sources provide high fluxes of $\mathrm{X}$-rays at higher energy than laboratory sources. These characteristics open the door not only to rapid data collection for high spatial-resolution structural studies, but also to time-resolved in situ/in operando measurements of functional materials under real conditions, thanks to high energy X-rays being able to penetrate operating environments. Furthermore, the use of high energy X-rays increases the region of reciprocal space which may be studied in an experiment, facilitating improved spatial resolution in total-scattering measurements.

Beamline P02.1 (PETRA III, DESY, Hamburg, Germany) is almost unique amongst high-resolution powder diffraction instruments in operating at a fixed energy of $60 \mathrm{keV}$ [1],[2]. This energy is particularly well suited to the collection of powder diffraction data for Rietveld refinement (especially for high- $Z$ materials, where absorption is a problem at lower energy) and for collection of total-scattering data (the high energy facilitating $\mathrm{Q}_{\mathrm{Max}}$ of at least $20 \AA^{-1}$ ), which may be interpreted by Pair Distribution Function (PDF) analysis. Currently two detectors are available on the beamline (a two-dimensional Perkin Elmer XRD1621 and an in-house developed 10-channel Multi-Analyser Detector), whilst additional devices are available from a shared detector pool (including a Dectris Pilatus3 CdTe 2M designed for high-energy data collection).

In addition to ambient temperature capillary measurements, P02.1 offers a range of standard sample environments (e.g. hot-air blower - RT-1100 K; cryostream - 90-500 K; cryostat $-10-300 \mathrm{~K}$ ) whilst a number of others are under development (e.g. low pressure gas adsorption cell) or are maintained by the PETRA III sample environment group. Recently we have also brought a sample changing robot into operation, with capacity for 300 samples; this device is compatible with the cryostream and hot-air blower environments. Furthermore, the enormous amount of free space around the diffractometer, compared to many other powder diffraction beamlines, allows easy integration of complicated sample environments which are brought to the beamline by our users. We report here the latest developments at P02.1 including planned changes to detector configurations, new sample environments, updates to the control software and possible remote access schemes.

References:

[1] Dippel A.-C. et al., Z. Anorg. Allg. Chem. 2014, 640, 3094.

[2] Dippel A.-C. et al., J. Synchrotron Radiat. 2015, 22, 675.

Keywords: synchrotron, automation, sample environments
MS44-P09

\section{Impact of tempering temperature on the properties of steel: An in-situ X-ray diffraction study}

Andreas Pein ${ }^{1}$, Manfrad Wiessner ${ }^{1}$

1. Anton Paar GmbH, Graz, Austria

email: andreas.pein@anton-paar.com

Material properties are strongly dependent on the structure and arrangement of the

atoms / molecules of which the material is comprised. For steel, different properties are required for the wide range of possible applications. Heat treatment (tempering) can be used to alter the microstructure and phases present within the steel and, therefore, change the material properties. In-situ X-ray diffraction measurements can be used to study these changes.

The mechanical properties of soft martensitic steels, which find use in a wide variety of applications, are strongly dependent on the microstructure which develops during heat treatment. After treatment, the steel consists of different phases; typically a combination of martensite, reverted or retained austenite, and carbide precipitates. Reverted austenite is the austenite which forms at sufficiently high temperatures during the tempering process.

Some superior properties found in these steels are high values in both fracture toughness and impact strength. These are caused by the microstructural arrangement of reverted austenite and martensite. It is therefore clear that the mechanical properties of the steel are dependent on the heat treatment as this influences the development of different phases and the microstructure within the steel.

In order to identify the effect of tempering temperature on the mechanical properties, in-situ high temperature X-ray diffraction is employed to study the microstructural changes occurring during tempering (1). This includes following the formation of different phases, as well as monitoring the dislocation densities; these can then be related to mechanical properties such as the impact strength and tensile strength. In this way, an improved understanding can be developed to optimize the tempering process in order to obtain steels with the desired properties.

References:

[1] Wiessner, M. et al. (2017) Materials Science \& Engineering, A682, 117-125

Keywords: in-situ X-ray diffraction, steel, copper tempering 